\title{
Medical physics publishing in a changing research environment: the Australasian Physical \& Engineering Sciences in Medicine 40th anniversary editorial
}

\author{
Scott B. Crowe ${ }^{1,2} \cdot$ Tanya Kairn $^{1,2}$
}

Published online: 29 November 2017

๑) Australasian College of Physical Scientists and Engineers in Medicine 2017

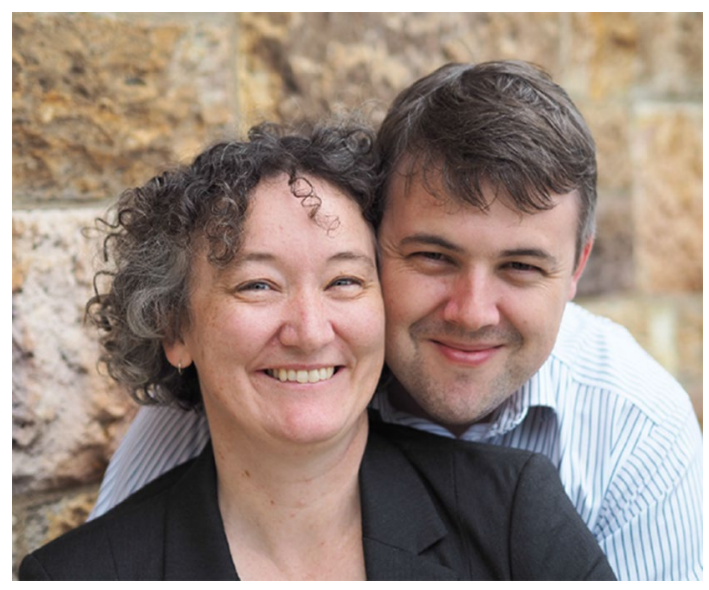

Australasian Physical \& Engineering Sciences in Medicine (APESM, or "the college journal") is now 40 years old, making it one of the oldest medical physics journals in the world [1]. In fact, the history of this journal stretches back an additional 18 years; APESM replaced the Australasian Bulletin of Medical Physics, which in turn replaced the Australasian Newsletter of Medical Physics, which was first published in December 1959. ${ }^{1}$

The history of APESM and the Australasian College of Physical Scientists in Medicine (ACPSEM) have been described in past editorials and commentaries that celebrated earlier anniversaries [2]. The 30th volume of APESM in particular presented a well-written history of APESM [2] in addition to 30 year retrospectives on medical physics in

Scott B. Crowe

sb.crowe@gmail.com

1 Cancer Care Services, Royal Brisbane \& Women's Hospital, Brisbane, Australia

2 Queensland University of Technology, Brisbane, Australia radiation oncology, radiology, nuclear medicine and radiation protection (some of which include fun photos of a few now-senior ACPSEM members) [3-6].

Since this history has been detailed so much and so well, we wanted to use the 40th anniversary editorial as an opportunity to consider the recent history of the college journal and to examine some of the things that have led to a rapid growth in the reach and influence of APESM in the last 10 years.

The growth of the reputation of APESM can be indicated, for example, by the impact factor. The impact factor, which has received recent commentaries from APESM editors Caon [7] and Trapp [8], is calculated as the number of citations of articles in a particular journal relative to the total number of articles published in that journal. APESM's impact factor has quickly increased from an initial value of 0.44 in 2012 to 1.17 in 2017 (above unity for the first time).

Past editorials [9-11] have highlighted changes to APESM production and distribution that have led to an increase in reach. Most obviously, partnering with a major international scientific publishing house (Springer International Publishing AG, Berlin, Germany) has resulted in dramatically increased subscription rates (electronic access to APESM articles is available via subscription packages at nearly 8000 libraries around the world), increased submission rates (Caon reported that submissions tripled between 2009 and 2010, the first year that the journal was published by Springer [10]), increased editorial discernment (the proportion of submissions rejected by APESM is currently increasing towards 70\%), and increased numbers of citations (described above). In 2016, manuscripts were received from authors in 40 countries, and reviewers were sourced from 41 countries [11]. Authors from around the world are now able to submit to APESM and cite APESM, because they are now able to see APESM.

\footnotetext{
1 The first issue of the college journal published in 1978 was labelled "Ser. No. 73" to indicate continuity of publication.
} 
The country producing the largest proportion of accepted manuscripts in APESM consistently remains Australia (at approximately $38 \%$ for the last 3 years) and New Zealand generally ranks fifth to seventh. When evaluating the improved reputation of APESM, therefore, the role of the increasing number and quality of submissions from this region should not be underestimated.

There have been changes in Australia and New Zealand's medical physics and engineering environment that have had important, indirect effects on the content and reputation of APESM. The following discussion will focus some of the changes specifically affecting radiation oncology medical physics, because that is our particular expertise. This discussion is expected to be interesting and useful to readers from other disciplines, although direct comparisons should be undertaken with caution.

\section{Adoption of new technologies}

Since the earliest reported delivery a modulated radiotherapy treatment in Australia and New Zealand in 2002 [12], the use of inverse planning has progressed to the point that most if not all centres in Australia and New Zealand are now treating with modulated techniques. The delivery of increasingly complex treatments to smaller targets has placed demands on physics time (with all treatments requiring patient-specific quality assurance testing) and has required vendors and individuals to develop more sophisticated one-, two- and threedimensional dosimeters, and to fabricate more specialised phantoms.

Investigations of these new technologies should be published, so that the worldwide medical physics community can gain the benefit of the hard work done by the few medical physicists who gain first access to the equipment. However, the well-known international medical physics journals seem particularly disinterested in such studies. For example, Medical Physics provides instructions to authors that advise against submitting "articles with a purely applied/clinical focus" [13], and Physics in Medicine and Biology has been known to reject papers identified by the editorial board as "very clinical" or "not containing new physics". ${ }^{2}$

APESM usually rejects commissioning reports on existing or previously published technologies, but this journal is the natural home for investigations of new technologies, or new ways to use existing technologies. Some of APESM's most-cited papers of the past 10 years specifically describe such investigations for film [14-16] and imaging modalities [17].

\footnotetext{
2 Personal communication, rejection letter from Physics in Medicine
} and Biology, 2015.
Australia and New Zealand also have a strong history of developing new technologies, and some important work in this area has recently been published by APESM. For example, Alnaghy et al.'s award-winning study of "gel-water" for MRI-linac dosimetry and imaging [18] describes a thorough investigation of a new phantom that could be used in Liverpool Hospital/University of Sydney's major MRI-linac development program.

For authors who are working on technology development, APESM currently seems to be an attractive place to describe and discuss early work and proof-of-concept studies. However, as the impact and reach of the journal is observed to increase, APESM will inevitably grow to also become the place to publish final and comprehensive descriptions of major technological development projects.

\section{Extension of services}

Three of the first twelve medical MV linear accelerators in the world were installed in Australia in 1956 and 1957, with one following soon after in New Zealand [19]. In the last ten years, we have continued to be "early adopters" of new technologies, including frameless stereotactic radiosurgery (Premion, 2007), TomoTherapy (Royal Brisbane and Women's Hospital, 2011), Cyberknife (Sir Charles Gairdner Hospital, 2014) and the Varian Halcyon (Radiation Oncology Centres Toowoomba, 2017).

Currently, Australia and New Zealand have some of the highest rates of MV treatment machines per capita in the Asia-Pacific region [20]. In the past 10 years, there has been a substantial increase in the number of MV treatment machines in Australia, some of which has been due to the opening up of new centres, especially in isolated regions not previously serviced by nearby radiation oncology facilities. For example, Darwin in 2010 (3028 km from Adelaide), Cairns in 2011 (1682 km from Brisbane), Bunbury in 2011 (169 km from Perth), Warrnambool in 2016 (257 km from Melbourne) and Burnie in 2016 (301 km from Hobart and $137 \mathrm{~km}$ from Launceston).

The number of accelerators in Australia is presented in Fig. 1, using data reported in the 1975 volume of The Radiographer [21-26] (assuming no decommissionings prior to 1970 ), as well as more-recent workforce reports [27, 28] and other documents [29-31].

These recent increases in the total number of radiotherapy centres, the number of isolated regional radiotherapy centres, the number of linacs in clinical use and the number of different types of (non-linac) treatment delivery systems all have the potential to lead to opportunities and challenges for physicists interested in research and in research publication. The number and heterogeneity of new treatment 


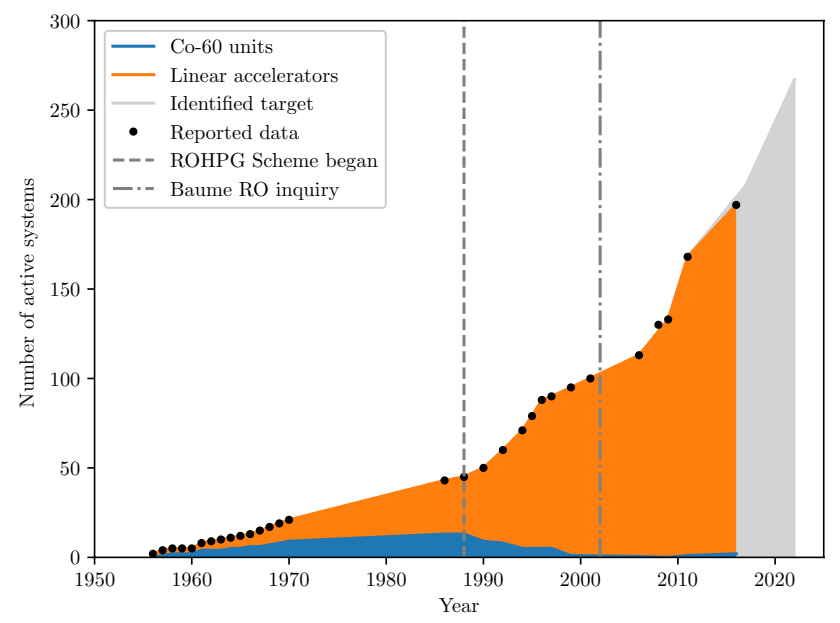

Fig. 1 Stacked area plot of number of MV teletherapy systems in Australia

delivery systems obviously gives us more opportunities to do exciting and original research work using new techniques and technologies. The fact that many of us are now working in isolated one- or two-physicist centres means that inter-departmental collaboration in our clinical work is now imperative, and clinical collaboration provides opportunities to discuss common problems and develop solutions that can and should be published as research, especially in APESM. However, these opportunities may amount to nothing more than frustration, if physics staffing levels do not keep pace with the ongoing expansion of treatment delivery.

\section{Clinical physics staffing}

Advertisements for radiation oncology medical physicist positions distributed by ACPSEM over the last 14 years have frequently included responsibility for "research and development", "research and training" or just "research" as part of the role description. ${ }^{3}$ Although research has been identified as an expected practise for clinical medical physicists [32], the ability to offer genuine research opportunities remains a key selling point, for centres attempting to recruit medical physicists. Participation in research has been linked to job satisfaction among medical physicists $[20,33]$ and the availability of physicists with research experience has been recognised as necessary for taking full advantage of available technology in the clinic [34].

Despite the obvious advantages to individual physicists (in terms of job satisfaction) and departments (in terms of

\footnotetext{
${ }^{3}$ Sourced from hardcopy job advertisements provided by Robert Fitchew.
}

national and international reputation) as well as to staff and patients at other centres around the world (due to physics time saved and treatment accuracy improved by the availability of relevant and helpful publications), research is often one of the first areas to be neglected when staff time is limited. This can happen on an instiutional level, when research positions are replaced by clinical positions or staff with specific research expertise are given full-time clinical responsibilities. It is also very likely to happen at an individual level, because the answer to the question "should I finish writing a manuscript that might help other physicists but is not necessarily due right now, or should I run a routine test that will allow a patient to receive their cancer treatment as soon as possible?" is likely to be obvious and unchanging, at every hour of every day until the manuscript is completely forgotten.

Even a small decrease in local physics staffing, or a small increase in clinical workload, can have large effects on research participation and research output. Consider this unverifiable anecdote: we know of a centre where, after the number of physics staff dropped by a quarter (due to staff leaving and not being replaced), the number of contributions to APESM decreased by two thirds.

The reason for this non-linear effect is obvious, when the research process is considered. Eight interrupted hours of research time might allow a physicist to plan and complete an important experimental study or write up part of a manuscript. Much, much less productivity is achievable when those eight hours are spread over a week or a month. It is extremely difficult to undertake productive research work, if that work must be fitted around an overwhelming clinical workload. In their analysis of trends in radiation oncology medical physics (ROMP) in the Asia-Pacific region, Kron, Healy and Ng noted that "many ROMPs are required to work overtime and not many find time for research" [35].

It should be understood that the pressures currently being faced by clinical medical physicists in Australia and New Zealand are not the result of an overall decrease in staff numbers, or even a decrease in the number of physicists per linac. Between 2008 and 2014, the number of radiation oncology medical physicists in Australia and New Zealand increased from 268 to 407 (52\%) [20]. In the same period, the number of treatment machines in Australia increased from 130 to approximately 185 (42\%) (see Fig. 1).

Rather, there has recently been an inarguable and rapid decrease in physics staffing levels relative to treatment complexity and the number of physics hours needed per patient treatment. Radiation oncology departments are currently responding to Medicare billing changes that incentivise the use of modulated radiotherapy and patient-specific quality assurance. Departments are also beginning to accommodate requests from patients for sophisticated techniques such as breath-hold-based breast treatments as well as staff 
enthusiasm for the adoption of potentially life-saving new ablative radiotherapy treatments. While the local implementation of all of these new and complex treatments (and all of the technology changes mentioned in previous sections) provide exciting opportunities for physics research, they all also require large amounts of physics time, which might make the completion of research projects and the publication of research outcomes impossible.

\section{Student numbers}

Some important APESM publications have come from postgraduate students because students are required, as part of their studies, to investigate research problems to a depth that is often unreachable by busy clinical physicists. APESM is a desirable place to publish student work, because it is seen and read by (and physically lands on the desks of) exactly the people from whom the students will be seeking employment. Compared to journals based overseas, there is also relatively easy access to editors and associate editors who can help resolve manuscript processing delays and who are sometimes willing to provide additional assistance in manuscript preparation for Australian or New Zealand students or registrars (just, before you ask for our time, remember that we are not paid to do this job).

The number of students studying medical physics has increased over the past 10 years. While accumulating data for our analysis of women's workforce and research participation [36], we found that the numbers of students enrolled in Masters and Doctoral Medical Physics programs at ACPSEM-accredited institutions were 179 and 98, respectively (35\% of whom were women). While this increase may be an admirable attempt by universities to supply the recognised demand for more physicists to staff the increasing numbers of radiation oncology centres [30], the supply of students with the qualifications needed to take up junior and training positions at radiation oncology clinics is currently exceeding the supply of entry-level positions.

An informal survey of heads of physics departments at radiation oncology departments that had advertised for registrars in 2015 and 2016 reported a mean of 41 suitable applicants per vacancy [37]; and gossip at the recent EPSM conference suggested that there have been advertisements attracting up to 80 applicants in 2017.

Our discipline should therefore be mindful that universities may be unintentionally doing a disservice to students by training so many more than are needed to fill available entrylevel positions. Alternatively, we may all be doing the community a disservice by not effectively lobbying to create the required numbers of entry-level positions that would allow former-students to work towards certification, to meet the ongoing need for experienced medical physicists to ensure the safe and accurate delivery of radiation treatments.

\section{ACPSEM TEAP}

Former-students who are outstanding enough and fortunate enough to be appointed into ACPSEM training, education and assessment programme (TEAP) registrar positions are required to have at least one first-authored manuscript (or two non-first-authored manuscripts) accepted for publication in a refereed journal, in addition to the examinations and the other TEAP requirements.

The TEAP publication requirement has produced challenges for APESM editors (increased numbers of submissions that need to be turned from clinical reports into publishable manuscripts, increased pressure to deliver reviews that are both fair and fast, and so on), but has ultimately been advantageous for the journal and our readers, allowing us to publish numerous high-quality papers that might not have been written otherwise. For example, two of APESM's four most cited papers of the last five years were written by TEAP registrars [17, 38].

This requirement provides a strong incentive for each registrar's clinical department to prioritise the completion of at least one publishable research project within the first 12 months of the registrars appointment, firstly because publication review can take a long time and secondly because the registrar will have other priorities in later years.

More generally, the TEAP publication requirement encourages accredited departments to maintain researchsupporting environments conducive to the timely completion of TEAP projects, which may also improve the job satisfaction of other physicists in those environments [33]. For isolated or under-staffed departments, this might require reaching out to build collaborations with centres that are well-staffed by physicists with research experience, thereby building a broader research culture which supports the links required for uninhibited mutual auditing and dosimetric intercomparisons.

\section{Conclusion}

While the dramatic increase in the reach of APESM has been primarily driven by the international access provided by our publisher, several factors in the local medical physics environment have indirectly led to growth of the reputation and influence of the journal, particularly in the radiation oncology medical physics area.

APESM is viewed as a desirable place to publish investigations of new technologies and new ways to use existing technologies and the release of so many new treatment 
delivery and dosimetry systems in the last decade has dramatically increased the number and importance of such publications.

The number, variety and geographical separation of new radiotherapy treatment systems in Australia and New Zealand has the potential to similarly lead to important publications, especially in APESM, if our current research culture can be preserved and enhanced through the growth of our medical physics workforce.

Maintaining and growing our workforce, by effectively lobbying for more entry-level positions and then giving our registrars a pathway to permanent employment at certification, is the best way to give ourselves the opportunity to build productive research collaborations and complete important research projects. The ability of medical physicists to effectively participate in research is fundamentally important for patient safety, not just because of the research work itself and the clinical application of its outcomes, but because of the collaborations (and potential dosimetric and cultural inter-comparison opportunities) arising from that research.

In other words, the Australian and New Zealand radiation oncology medical physics research culture might be the "canary in the coalmine", indicating the safety of radiation oncology practises in this region. And publications in APESM, the official journal of the ACPSEM, might be the "canary in the coalmine's canary in the coalmine", indicating the healthiness of Australia and New Zealands radiation oncology medical physics research culture.

Today, APESM is a very healthy canary, but we all know there are hazards ahead. And we have to provide the best help and support we can for the next generation of APESM authors and reviewers and editors, because they will be better than us and, given the opportunity, they will grow and develop this journal (and the medical physics discipline) in ways we cannot even imagine at this point. After all, APESM is only 40 years old.

Acknowledgements The authors wish to thank Lyn Oliver for supplying information regarding the introduction of MV therapy systems in Australia, Robert Fitchew for supplying back issues of APESM (and its predecessors) and providing access to his personal archive of ACPSEM documentation, Nigel Middlebrook for providing information on the growth of radiotherapy services in Western Australia, and Martin Caon for supplying back issues of APESM as well as Spinger's 2016 "publisher's report" on APESM.

\section{References}

1. Caon M (2016) There are too many medical physics journals! Australas Phys Eng Sci Med 39(4):813-816

2. Clarke K (2007) An historical perspective of the APESM. Australas Phys Eng Sci Med 30(1):xiv-Xv
3. Oliver LD (2007) Thirty year celebration of journal publications on radiation oncology medical physics. Australas Phys Eng Sci Med 30(1):1-12

4. Heggie JCP (2007) Technical developments in radiology in Australasia dating from 1977. Australas Phys Eng Sci Med 30(3):160-177

5. Walker BM (2007) Thirty year celebration of the contribution of nuclear medicine physicists in Australia. Australas Phys Eng Sci Med 30(4):239-251

6. Smart RC (2007) Radiation protection in Australia: a thirty year perspective. Australas Phys Eng Sci Med 30(3):155-159

7. Caon M (2017) Gaming the impact factor: where who cites what, whom and when. Australas Phys Eng Sci Med 40(2):273-276

8. Trapp J (2016) Web of Science, Scopus, and Google Scholar citation rates: a case study of medical physics and biomedical engineering: what gets cited and what doesnt? Australas Phys Eng Sci Med 39(4):812-823

9. Caon M (2009) State of the journal, APESM statistics 20052008. Australas Phys Eng Sci Med 32(1):xiii-xiv

10. Caon M (2013) March 2013 editorial: operational statistics for the APESM journal (Jan 2012-Feb 2013). Australas Phys Eng Sci Med 36(1):5-7

11. Caon M (2017) Operational statistics for the APESM journal (2014-2016). Australas Phys Eng Sci Med 40(3):487-489

12. Corry J, Hope G, Cramb J, Smylie J, Joon DL, Towns S, Archer P, Henkul Z, Wills J (2002) Intensity-modulated radiation therapy: first reported treatment in Australasia. J Med Imaging Radiat Oncol 46(3):285-289

13. Online W (2017) Medical physics: author guidelines. http://aapm.onlinelibrary.wiley.com/hub/journal/10.1002/ (ISSN)2473-4209/about/author-guidelines.html. Accessed 1 Nov 2017

14. Butson MJ, Cheung T, Yu PKN, Alnawaf H (2009) Dose and absorption spectra response of EBT2 Gafchromic film to high energy X-rays. Australas Phys Eng Sci Med 32(4):196-202

15. Aland T, Kairn T, Kenny J (2011) Evaluation of a Gafchromic EBT film dosimetry system for radiotherapy quality assurance. Australas Phys Eng Sci Med 34(2):251-260

16. Kairn T, Hardcastle N, Kenny J, Meldrum R, Tomé W, Aland T (2011) Ebt2 radiochromic film for quality assurance of complex IMRT treatments of the prostate: micro-collimated IMRT, RapidArc, and TomoTherapy. Australas Phys Eng Sci Med 34(3):333

17. Walker A, Liney G, Metcalfe P, Holloway L (2014) MRI distortion: considerations for MRI based radiotherapy treatment planning. Australas Phys Eng Sci Med 37:103-113

18. Alnaghy SJ, Gargett M, Liney G, Petasecca M, Begg J, Espinoza A, Newall MK, Duncan M, Holloway L, Lerch MLF, Lazea M, Rosenfeld AB, Metcalfe P (2016) Initial experiments with gelwater: towards MRI-linac dosimetry and imaging. Australas Phys Eng Sci Med 39(4):921-932

19. Thwaites DI, Tuohy JB (2006) Back to the future: the history and development of the clinical linear accelerator. Phys Med Biol 51(13):R343-R362

20. Kron T, Azhari HA, Voon EO, Cheung KY, Ravindran P, Soejoko D, Inamura K, Han Y, Ung NM, TsedenIsh B, Win UM, Srivastava R, Marsh S, Farrukh S, Rodriguez L, Kuo M, Baggarley S, DilipKumara AH, Lee CC, Kisanachinda A, Nguyen XC, Ng KH (2015) Medical physics aspects of cancer care in the Asia Pacific region: 2014 survey results. Australas Phys Eng Sci Med 38(3):493-501

21. Bailey MM, Chambers JMS, Manny ML (1975) Down the maze: radiotherapy in New South Wales. Radiographer 22(1):8-11

22. Graham R (1975) Then to now: Radiotherapy in Victoria. Radiographer 22(1):37

23. Poller J (1975) Radiotherapy in Western Australia 1902-1974. Radiographer 25:24-25 
24. Tweddell J (1975) The history of radiotherapy in Queensland. Radiographer 22(1):4-7

25. Wilson M (1975) Radiotherapy in Tasmania a tale of two cities. Radiographer 22(1):16-20

26. Woodger EG (1975) South Australia radiotherapy over 45 years. Radiographer 22(1):21-3

27. Wigg DR (1988) Radiation oncology in Australia: an increasing crisis. J Med Imaging Radiat Oncol 32(1):24-37

28. Wigg DR, Morgan GW (2001) Radiation oncology in Australia: workforce, workloads and equipment 1986-1999. J Med Imaging Radiat Oncol 45(2):146-169

29. Oliver L (2009) The history of medical physics in Australian radiotherapy. J Med Imaging Radiol Oncol 53:A95

30. Radiation Oncology Tripartite Committee (2012) Planning for the best: tripartite national strategic plan for radiation oncology 2012-2022. The Royal Australian and New Zealand College of Radiologists, Melbourne

31. MPConsulting (2016) Review of the Radiation Oncology Health Program Grants (ROHPG) Scheme. The Department of Health, London

32. Round W, Stefanoyiannis A, Ng K, Rodriguez L, Thayalan K, Han Y, Tang F, Fukuda S, Srivastava R, Krisanachinda A et al
(2015) AFOMP Policy No 5: career progression for clinical medical physicists in AFOMP countries. Australas Phys Eng Sci Med 38(2):217-221

33. Ebert MA, Halkett GKB, Berg M, Cutt D, Davis M, Hegney D, House M, Krawiec M, Kearvell R, Lester L, Maresse S, McLoone P, McKay J (2017) An assessment of radiation oncology medical physicists perspectives on undertaking research. Australas Phys Eng Sci Med 40(1):173-180

34. Suchowerska N (2010) The international arena of medical physics: where is Australasia? Australas Phys Eng Sci Med 33(2):125-127

35. Kron T, Healy B, Ng KH (2016) Surveying trends in radiation oncology medical physics in the Asia Pacific Region. Phys Med 32(7):883-888

36. Crowe SB, Kairn T (2016) Women in medical physics: a preliminary analysis of workforce and research participation in Australia and New Zealand. Australas Phys Eng Sci Med 39(2):525-532

37. Crowe SB (2017) Advice for medical physics job seekers. http:// sbcrowe.net/advice-medical-physics-job-seekers (2016). Accessed 1 Nov 2017

38. Moylan R, Aland T, Kairn T (2013) Dosimetric accuracy of Gafchromic EBT2 and EBT3 film for in vivo dosimetry. Australas Phys Eng Sci Med 36(3):331-337 